\title{
4D Einstein-Gauss-Bonnet gravity coupled with nonlinear electrodynamics
}

\author{
S. I. Kruglov ${ }^{1}$ \\ Department of Physics, University of Toronto, \\ 60 St. Georges St., Toronto, ON M5S 1A\%, Canada \\ Department of Chemical and Physical Sciences, University of Toronto, \\ 3359 Mississauga Road North, Mississauga, Ontario L5L 1C6, Canada
}

\begin{abstract}
An exact spherically symmetric and magnetically charged black hole solution in 4D Einstein-Gauss-Bonnet gravity coupled to nonlinear electrodynamics (NED) is obtained. The NED Lagrangian is given by $\mathcal{L}_{N E D}=-\mathcal{F} /(1+\sqrt[4]{2 \beta \mathcal{F}})$, where $\mathcal{F}$ is the field invariant. We study the thermodynamics calculating the Hawking temperature and the heat capacity of the black hole. The phase transitions take place when the Hawking temperature has an extremum and the heat capacity is singular. We demonstrate that black holes are thermodynamically stable in some range of event horizon radii where the heat capacity is positive.
\end{abstract}

\section{Introduction}

The heterotic string theory at the low energy limit gives models of gravity with higher order curvature terms in the action [1]. Therefore, it is interesting to investigate a modified Einstein General Relativity (GR) including higher order curvature terms. Probably, such deformed GR can describe gravity in the strong gravity regime where quantum corrections are important. An interesting modified GR with higher order curvature terms is the Lovelock gravity in higher dimensions leading to the second order field equations. The particular case of the Lovelock theory of gravity is the Gauss-Bonnet (GB) gravity resulting to non-trivial dynamics. In four dimensions such theory (4D EGB) includes the Einstein-Hilbert action and the GB term which is a topological invariant and, as a result, it does not contribute to the gravitational dynamics. But Glavan and Lin [2] shown that if the coupling constant

\footnotetext{
${ }^{1}$ E-mail: serguei.krouglov@utoronto.ca
} 
$\alpha$, which can be considered as the inverse of the string tension, is re-scaled by $\alpha /(D-4)$, in the limit $D \rightarrow 4$, the theory yields a non-trivial dynamics without singularities. This approach was also discussed in [3], [4]. The 4D EGB theory, being a classical modified gravity, is free from the Ostrogradsky instability and conserves the number of degrees of freedom. The static spherically symmetric BH solution for the EGB gravity was obtained in [5] and the GB term can be considered as a quantum correction to GR. It worth noting that the BH solution gives a repulsive gravitational force at short distances. Similar solution was obtained in the gravity theory with quantum corrections [6]. Recently, the 4D EGB gravity theory attracted extensive attention (see, for example, [7]- [17]). The BH solutions in the 4D EGB model coupled to nonlinear electrodynamics (NED) were studied in [18]-[28].

In this paper, we obtain a $\mathrm{BH}$ solution in the 4D EGB model coupled to a particular case of NED proposed in [29]. This model of NED is a simple generalisation of linear Maxwell electrodynamics and allows us to get formulas expressed through elementary functions.

The paper is organized as follows: In Sec. 2, we obtain spherically symmetric solutions in the 4D EGB model coupled to NED. Depending on the model parameters, the metric function can have one (extreme) or two $\mathrm{BH}$ horizons or no horizons corresponding to particle-like solution. In Sec. 3 we calculate the Hawking temperature and the heat capacity. It was shown that the BHs are thermodynamically stable in some range of event horizon radii. In Sec. 4 we calculated the $\mathrm{BH}$ shadow within our model. Section 5 is devoted to a conclusion.

\section{4D EGB model coupled with NED}

The EGB gravity action in D-dimensions coupled to NED is given by

$$
I=\int d^{D} x \sqrt{-g}\left[\frac{1}{16 \pi G}\left(R+\frac{\alpha}{D-4} \mathcal{L}_{G B}\right)+\mathcal{L}_{N E D}\right]
$$

where $\alpha$ possesses the dimension of (length) ${ }^{2}$ and the particular NED Lagrangian, proposed in $[29]^{2}$, is given by

$$
\mathcal{L}_{N E D}=-\frac{\mathcal{F}}{1+\sqrt[4]{2 \beta \mathcal{F}}}
$$

\footnotetext{
${ }^{2}$ For a convenience we substituted $\beta$ in $[29]$ by $2 \beta$.
} 
where the parameter $\beta(\beta \geq 0)$ has the dimension of (length $)^{4}, \mathcal{F}=(1 / 4) F_{\mu \nu} F^{\mu \nu}=$ $\left(B^{2}-E^{2}\right) / 2, F_{\mu \nu}=\partial_{\mu} A_{\nu}-\partial_{\nu} A_{\mu}$ is the field tensor. The model of NED (2) is of interest because of its simplicity. The GB Lagrangian reads

$$
\mathcal{L}_{G B}=R^{\mu \nu \alpha \beta} R_{\mu \nu \alpha \beta}-4 R^{\mu \nu} R_{\mu \nu}+R^{2} .
$$

The variation of the action (1) with respect to the metric gives the field equations

$$
R_{\mu \nu}-\frac{1}{2} g_{\mu \nu} R+\frac{\alpha}{D-4} H_{\mu \nu}=-8 \pi G T_{\mu \nu}
$$

where

$$
H_{\mu \nu}=2\left(R R_{\mu \nu}-2 R_{\mu \alpha} R_{\nu}^{\alpha}-2 R_{\mu \alpha \nu \beta} R^{\alpha \beta}-R_{\mu \alpha \beta \gamma} R_{\nu}^{\alpha \beta \gamma}\right)-\frac{1}{2} \mathcal{L}_{G B} g_{\mu \nu} .
$$

The symmetrical stress-energy tensor of the NED (2) [29] is given by

$$
T_{\mu \nu}=-\frac{(4+3 \sqrt[4]{2 \beta \mathcal{F}}) F_{\mu}{ }^{\alpha} F_{\nu \alpha}}{4(1+\sqrt[4]{2 \beta \mathcal{F}})^{2}}-g_{\mu \nu} \mathcal{L}_{N E D}
$$

The GR coupled with NED, having the Maxwell limit $\mathcal{F} \rightarrow 0$, does not admit a static, spherically symmetric solution with a regular center and a nonzero electric charge [?]. Thus, to have a regular solution as $r \rightarrow 0$ we study a magnetic BH. The magnetic energy density, found from Eq. (6), is

$$
\rho_{M}=T_{t}{ }^{t}=\frac{B^{2}}{2\left(\beta^{1 / 4} \sqrt{B}+1\right)}=\frac{q_{m}^{2}}{2 r^{3}\left(r+\sqrt{q_{m}} \beta^{1 / 4}\right)},
$$

where $q_{m}$ is a magnetic charge, $\mathcal{F}=q_{m}^{2} /\left(2 r^{4}\right)$. The $t t$ component of the field equation (4), at the limit $D \rightarrow 4$, gives

$$
r\left(2 \alpha f(r)-r^{2}-2 \alpha\right) f^{\prime}(r)-\left(r^{2}+\alpha f(r)-2 \alpha\right) f(r)+r^{2}-\alpha=\frac{q_{m}^{2} r G}{r+\sqrt{q_{m}} \beta^{1 / 4}} .
$$

The static and spherically symmetric line element squared is given by

$$
d s^{2}=-f(r) d t^{2}+\frac{1}{f(r)} d r^{2}+r^{2}\left(d \vartheta^{2}+\sin ^{2} \vartheta d \phi^{2}\right) .
$$

The solution to Eq. (8) gives the metric function $f(r)$.

$$
f(r)=1+\frac{r^{2}}{2 \alpha}\left(1-\sqrt{1+\frac{8 M \alpha G}{r^{3}}+\frac{4 \alpha q_{m}^{3 / 2} G}{\beta^{1 / 4} r^{3}} \ln \left(\frac{r}{r+\sqrt[4]{\beta q_{m}^{2}}}\right)}\right)
$$


Introducing the dimension-less variable $x=r / \sqrt[4]{\beta q_{m}^{2}}$, Eq. (10) becomes

$$
f(x)=1+c x^{2}-c \sqrt{x^{4}+x\left(a+b \ln \left(\frac{x}{x+1}\right)\right)},
$$

where the dimension-less parameters are

$$
a=\frac{8 M \alpha G}{\beta^{3 / 4} q_{m}^{3 / 2}}, \quad b=\frac{4 \alpha G}{\beta}, \quad c=\frac{\sqrt{\beta} q_{m}}{2 \alpha},
$$

where $8 M \alpha G$ is the constant of integration and $M$ is the $\mathrm{BH}$ mass. We has chosen the sign minus in the front of the square root in Eqs. (10) and (11) to have the stable BH (see [5]). The solution to Eq. (8) at $\beta=0$, making use of the limit $\beta \rightarrow 0$ in Eq. (8) before the integration, becomes [7]

$$
f(r)=1+\frac{r^{2}}{2 \alpha}\left(1-\sqrt{1+\frac{8 M \alpha G}{r^{3}}-\frac{4 \alpha q_{m}^{2} G}{r^{4}}}\right)
$$

It should be stressed that the limit $r \rightarrow 0$ in Eq. (13) leads to the nonphysical complex value of the metric function $f(r)$. At the same time, the limit $r \rightarrow 0$ in Eqs. (10) and (11) gives the necessary value $f(0)=1$. The plot of the function (11) is depicted in Fig. 1. Figure 1 shows that there can be one extreme horizon or two horizons, or not horizons with particle-like solution.

\section{The $\mathrm{BH}$ thermodynamics}

The Hawking temperature is given by

$$
T_{H}\left(r_{+}\right)=\frac{f^{\prime}\left(r_{+}\right)}{4 \pi}
$$

where $r_{+}$is the event horizon radius $\left(f\left(r_{+}\right)=0\right)$. With the help of Eq. (11) $\left(x=r / \sqrt[4]{\beta q_{m}^{2}}\right)$ we find the Hawking temperature

$$
T_{H}\left(x_{+}\right)=\frac{c}{4 \pi \sqrt[4]{\beta q_{m}^{2}}}\left(2 x_{+}-\frac{4 x_{+}^{3}+a+b \ln \left(x_{+} /\left(x_{+}+1\right)\right)+b /\left(1+x_{+}\right)}{2 \sqrt{x_{+}^{4}+a x_{+}+b x_{+} \ln \left(x_{+} /\left(x_{+}+1\right)\right)}}\right) .
$$




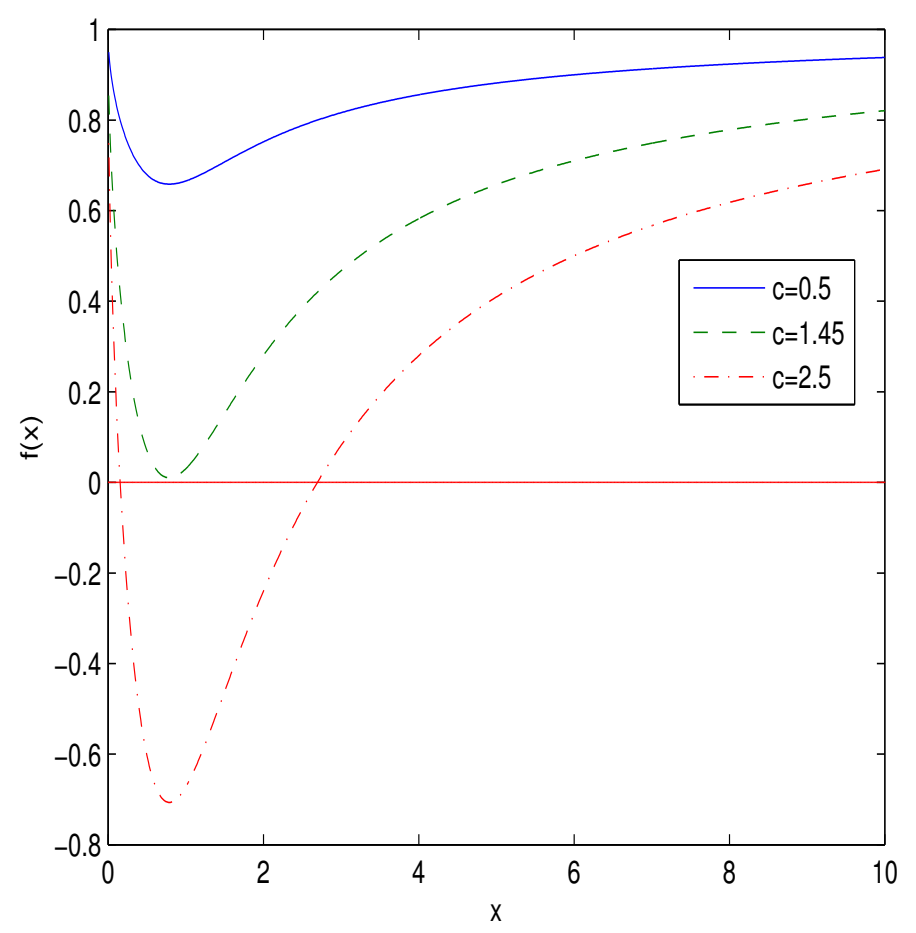

Figure 1: The plot of the function $f(x)$ for $a=c=1$.

The plot of the dimension-less function $T_{H}\left(x_{+}\right) \sqrt[4]{\beta q_{m}^{2}}$ is given in Fig. 2 . According to Fig. 2 the Hawking temperature is positive in some range of the event of horizon radii $x_{+}$. When the Hawking temperature is negative the $\mathrm{BH}$ does not exist. Making use of equation $f\left(x_{+}\right)=0$ we obtain the $\mathrm{BH}$ gravitational mass

$$
M\left(x_{+}\right)=\frac{\beta^{3 / 4} q_{m}^{3 / 2}}{8 \alpha G}\left(\frac{\left(1+c x_{+}^{2}\right)^{2}}{c^{2} x_{+}}-x_{+}^{3}-b \ln \left(\frac{x_{+}}{x_{+}+1}\right)\right) .
$$

The first law of $\mathrm{BH}$ thermodynamics is

$$
d M\left(x_{+}\right)=T_{H}\left(x_{+}\right) d S+\phi d q .
$$

Making use of Eq. (17) we find the entropy at the constant charge

$$
S=\int \frac{d M\left(x_{+}\right)}{T_{H}\left(x_{+}\right)}=\int \frac{1}{T_{H}\left(x_{+}\right)} \frac{\partial M\left(x_{+}\right)}{\partial x_{+}} d x_{+} .
$$




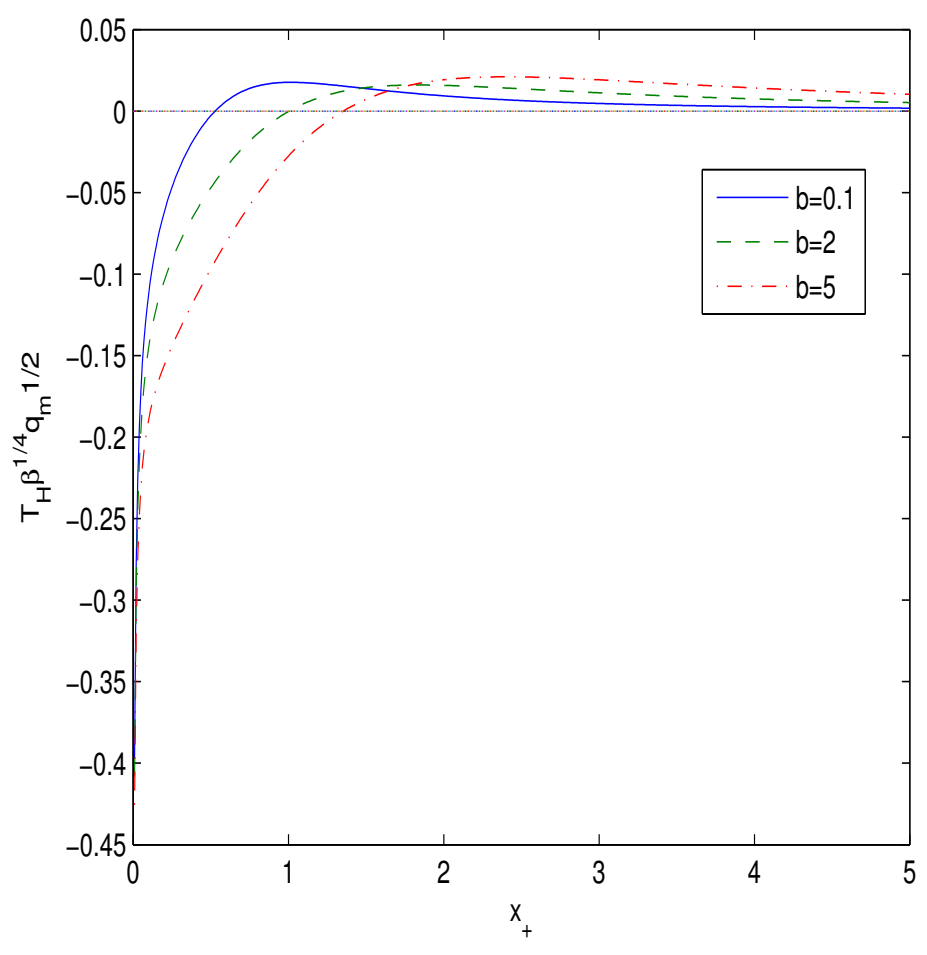

Figure 2: The plot of the function $T_{H}\left(x_{+}\right) \sqrt[4]{\beta q_{m}^{2}}$ at $a=c=1$.

With the help of Eq. (18) one obtains the heat capacity

$$
C_{q}\left(x_{+}\right)=T_{H}\left(\frac{\partial S}{\partial T_{H}}\right)_{q}=\frac{\partial M\left(x_{+}\right)}{\partial T_{H}\left(x_{+}\right)}=\frac{\partial M\left(x_{+}\right) / \partial x_{+}}{\partial T_{H}\left(x_{+}\right) / \partial x_{+}} .
$$

From Eq. (16) we find

$$
\begin{aligned}
\frac{\partial M\left(x_{+}\right)}{\partial x_{+}}=\frac{\beta^{3 / 4} q_{m}^{3 / 2}}{8 \alpha G}\left(\frac{2 c x_{+}^{2}-1}{c^{2} x_{+}^{2}}-\frac{b}{x_{+}\left(1+x_{+}\right)}\right) \\
\frac{\partial T_{H}\left(x_{+}\right)}{\partial x_{+}}=\frac{c}{4 \pi \sqrt[4]{\beta q_{m}^{2}}}\left(2-\frac{12 x_{+}^{2}+2 b /\left(x_{+}\left(1+x_{+}\right)\right)-b x_{+}\left(1+2 x_{+}\right) /\left(x_{+}^{2}\left(1+x_{+}\right)^{2}\right)}{2 \sqrt{x_{+}^{4}+a x_{+}+b x_{+} \ln \left(x_{+} /\left(x_{+}+1\right)\right)}}\right. \\
\left.+\frac{\left(4 x_{+}^{3}+a+b \ln \left(x_{+} /\left(x_{+}+1\right)\right)+b /\left(1+x_{+}\right)\right)^{2}}{4\left(x_{+}^{4}+a x_{+}+b x_{+} \ln \left(x_{+} /\left(x_{+}+1\right)\right)\right)^{3 / 2}}\right)
\end{aligned}
$$


In accordance with Eq. (19) the heat capacity has a singularity if the Hawking temperature possesses an extremum, $\partial T_{H}\left(x_{+}\right) / \partial x_{+}=0$. Taking into account Eqs. (19), (20) and (21) we plotted the heat capacity as a function of the variable $x_{+}$in Fig. 3. According to Fig. 3 the $\mathrm{BH}$ is locally stable in

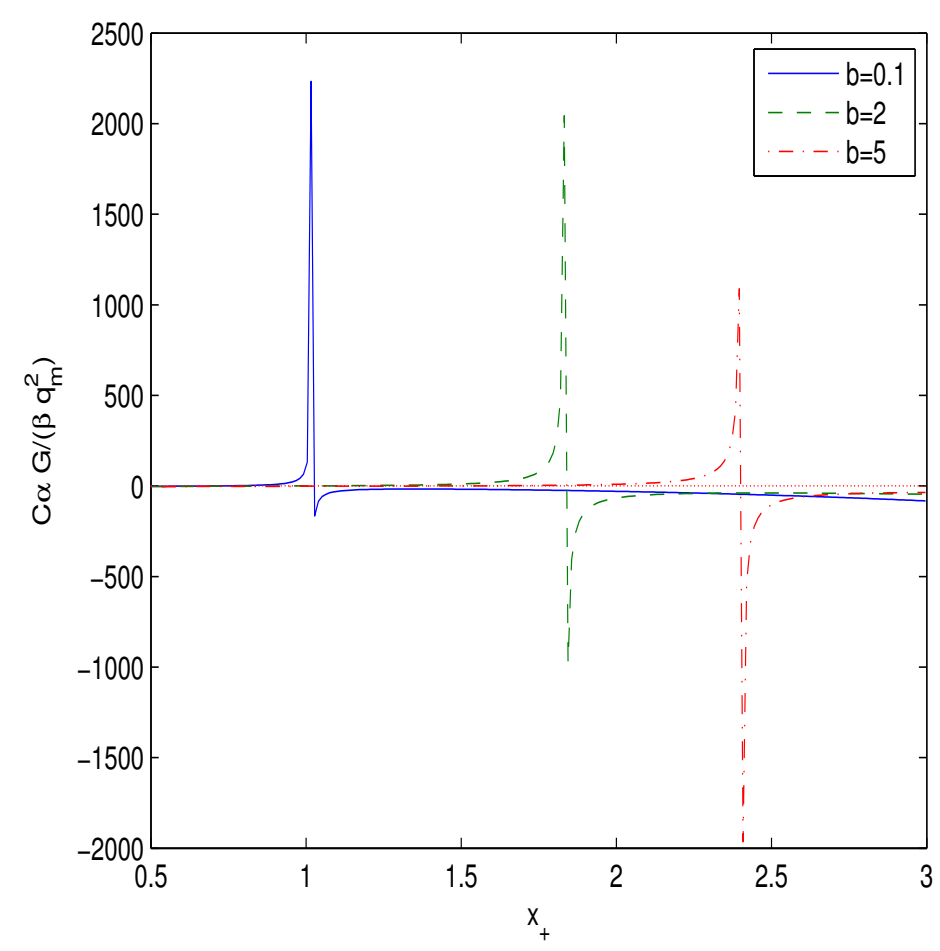

Figure 3: The plot of the function $C_{q}\left(x_{+}\right) \alpha G /\left(\beta q_{m}^{2}\right)$ at $a=c=1, b=2$.

some interval of the horizon radii where the heat capacity is positive. The singularity in the heat capacity occurs in the radii where the Hawking temperature possesses the extremum. In these points the second-order phase transition takes place.

\section{The black hole shadow}

The spherically symmetric BH shadow represents a black circular disk which is due to the gravitational lensing of light. The Event Horizon Telescope 
collaboration received the first image of the super-massive $\mathrm{M} 87^{*} \mathrm{BH}$ located in the center of the elliptical galaxy Messier 87 [31]. Firstly, the shadow of a neutral Schwarzschild $\mathrm{BH}$, described by the mass and the position of the observer, was studied in [32]. Here, we investigate the $\mathrm{BH}$ shadow within our model of 4D EGB coupled to NED (2). We will employ the Hamiltonian approach for the description of the photon motion in the static spherically symmetric space-time. We consider the photons moving in the equatorial plane with $\vartheta=\pi / 2$. Making use of the Hamilton-Jacobi method for null curves the photon motion is governed by the equation [33]

$$
H=\frac{1}{2} g^{\mu \nu} p_{\mu} p_{\nu}=\frac{1}{2}\left(\frac{L^{2}}{r^{2}}-\frac{E^{2}}{f(r)}+f(r) p_{r}^{2}\right)=0,
$$

where $p_{\mu}$ are photon momenta, $\dot{r}=\partial H / p_{r}, E=-p_{t}$ and $L=p_{\phi}$ are the energy and angular momentum of the photon (constants of motion). Equation (22) can be written as

$$
V+\dot{r}^{2}=0, \quad V=f(r)\left(\frac{L^{2}}{r^{2}}-\frac{E^{2}}{f(r)}\right) .
$$

The circular orbit radius $r_{p}$ of the photon can be obtained by solving the equation $V\left(r_{p}\right)=V^{\prime}(r)_{\mid r=r_{p}}=0$, where the prime denotes the derivative with respect to the argument. Thus, we find

$$
\xi \equiv \frac{L}{E}=\frac{r_{p}}{\sqrt{f\left(r_{p}\right)}}, \quad f^{\prime}\left(r_{p}\right) r_{p}-2 f\left(r_{p}\right)=0,
$$

where $\xi$ is the impact parameter. We use the numerical method to solve Eq. (22) for obtaining the radius of the photon sphere. The shadow radius $r_{s}$ of the $\mathrm{BH}$ observed by a static observer in the position $r_{0}$ is given by [33]

$$
r_{s}=r_{p} \sqrt{\frac{f\left(r_{0}\right)}{f\left(r_{p}\right)}}
$$

For a distant observer, $r_{0} \rightarrow \infty$, we can put $f\left(r_{0}\right)=1$ in Eq. (25) and the impact parameter $\varsigma=r_{s}$. The event horizon radius is defined by the biggest root of the equation $f\left(r_{h}\right)=0$. From Eq. (11) and $f\left(r_{h}\right)=0$ we obtain the dependence of the parameters $a$ and $b$ on the horizon radii at $c=1$

$$
a=\frac{1+2 x_{h}^{2}-x_{h} b \ln \left(x_{h} /\left(x_{h}+1\right)\right)}{x_{h}} \quad c=1,
$$




$$
b=\frac{1+2 x_{h}^{2}-x_{h} a}{x_{h} \ln \left(x_{h} /\left(x_{h}+1\right)\right)} \quad c=1,
$$

The plots of the functions (26) at $b=1,2,3$ and (27) at $a=1,2,3$ are depicted in Fig. 4 and 5, respectively. Figure 4 shows that when the

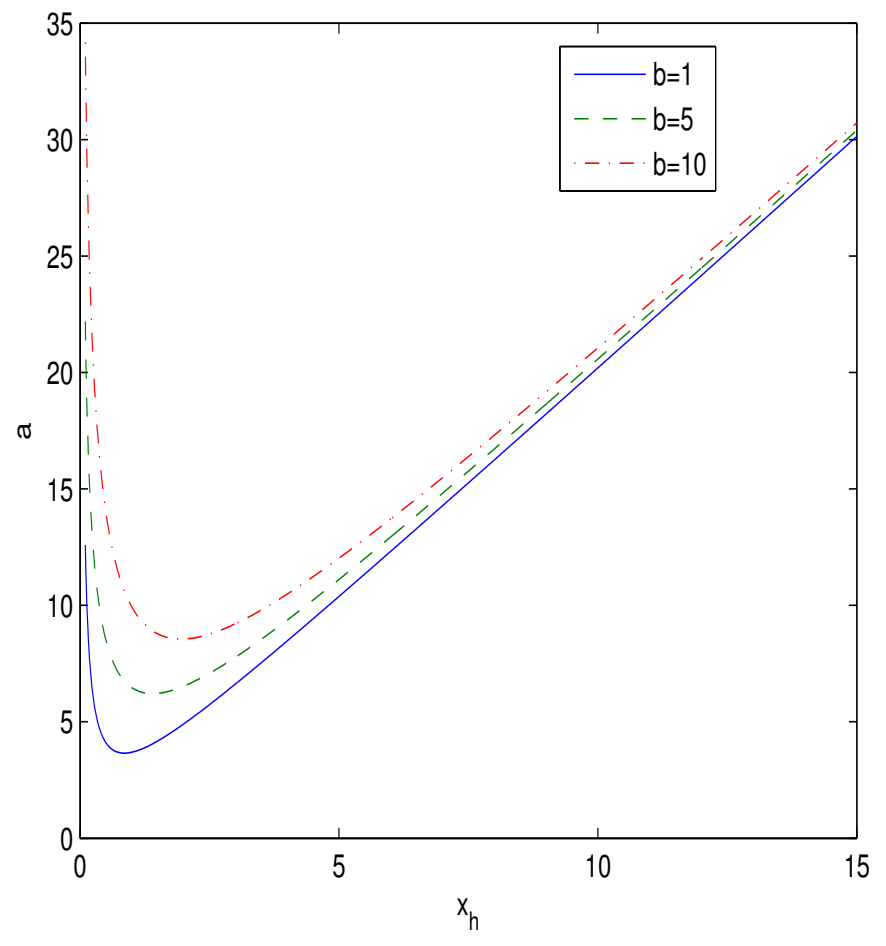

Figure 4: The plot of the function $a\left(x_{h}\right)$ at $c=1, b=1,5,10$.

parameter $b$ increases, the event horizon (the biggest root of equation $f\left(x_{h}\right)=$ 0 ) decreases. According to Fig. 5 if the parameter $a$ increases, the event horizon increases.

The photon sphere radii $\left(x_{p}\right)$, the event horizon radii $\left(x_{h}\right)$, and the shadow radii $\left(x_{s}\right)$ for some parameters $a, b$, and $c$, found from Eqs. (24) and (25), are presented in Table 1 (in terms of dimensionless variables). In according with Table 1 , when increasing the parameter $b$ at fixed $a$ and $c$, the shadow radius $x_{s}$ decreases. 


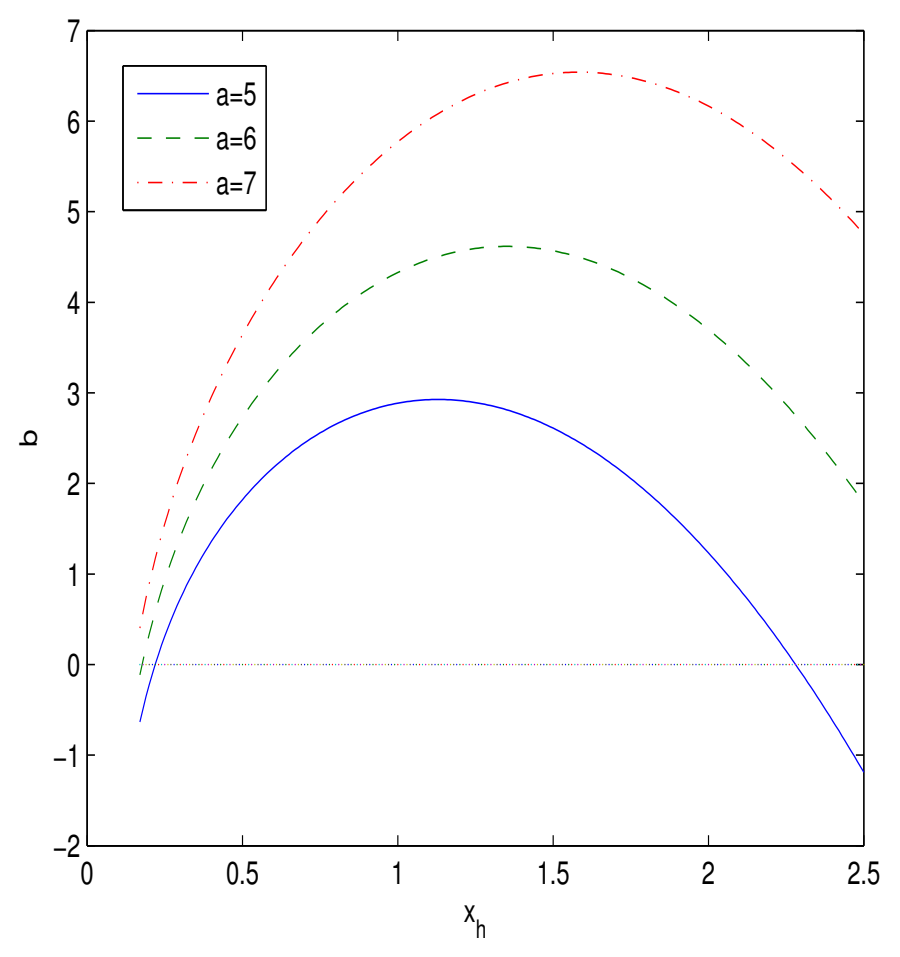

Figure 5: The plot of the function $b\left(x_{h}\right)$ at $c=1, a=5,6,7$.

\section{Conclusion}

The exact spherically symmetric and magnetically charged $\mathrm{BH}$ solution in 4D EGB gravity with NED, proposed in [29], was obtained. It was shown that the $\mathrm{BH}$ can have two horizons or one extreme horizon, or not horizons corresponding to particle-like solution, depending on the model parameters $\left(\alpha, \beta, q_{m}\right.$ and $\left.M\right)$. At the limit $r \rightarrow 0$ in the metric function has the reasonable value $f(0)=1$ while in the pure $4 \mathrm{D}$ EGB theory this limit gives the non-physical value of $f(0)$. The BH Hawking temperature and the heat capacity were calculated. We demonstrated that the phase transitions occur when the heat capacity has the singularity and the Hawking temperature possesses an extremum. The BHs are thermodynamically stable in some range of event horizon radii where the heat capacity is positive. We calculated the $\mathrm{BH}$ shadow within our model for some model parameters. 
Table 1: The event horizon radii, photon sphere and shadow radii for $a=5$, $\mathrm{c}=1$

\begin{tabular}{cccccccccc}
\hline$b$ & 1.5 & 1.7 & 1.8 & 2 & 2.2 & 2.3 & 2.4 & 2.5 & 2.6 \\
\hline$x_{h}$ & 1.93 & 1.87 & 1.84 & 1.77 & 1.69 & 1.65 & 1.61 & 1.56 & 1.51 \\
\hline$x_{p}$ & 3.12 & 3.05 & 3.01 & 2.94 & 2.86 & 2.82 & 2.77 & 2.73 & 2.68 \\
\hline$x_{s}$ & 5.78 & 5.70 & 5.65 & 5.56 & 5.47 & 5.42 & 5.37 & 5.32 & 5.26
\end{tabular}

\section{References}

[1] D. J. Gross and E. Witten, Nucl. Phys. B 277, 1 (1986); D. J. Gross and J. H. Sloan, Nucl. Phys. B 291, 41 (1987); R. R. Metsaev and A. A. Tseytlin, Phys. Lett. B 191, 354 (1987); B. Zwiebach, Phys. Lett. B 156, 315 (1985); R. R. Metsaev and A. A. Tseytlin, Nucl. Phys. B 293, 385 (1987).

[2] D. Glavan and C. Lin, Phys. Rev. Lett. 124, 081301 (2020).

[3] M. Gurses, T. C. Sisman, and B. Tekin, Phys. Rev. Lett. 125, 149001 (2020).

[4] M. Gurses, T. C. Sisman, and B. Tekin, Eur. Phys. J. C 80, 647 (2020).

[5] D. G. Boulware and S. Deser, Phys. Rev. Lett. 55, 2656 (1985); J. T. Wheeler, Nucl. Phys. B 268, 737 (1986); R.C. Myers and J. Z. Simon, Phys. Rev. D 38, 2434 (1988).

[6] G. Cognola, R. Myrzakulov, L. Sebastiani, and S. Zerbini, Phys. Rev. D 88, 024006 (2013),

[7] P. G. S. Fernandes, Phys. Lett. B 805135468 (2020).

[8] S. G. Ghosh and S. D. Maharaj, Phys. Dark Univ. 30, 100687 (2020).

[9] R. Kumar and S. G. Ghosh, JCAP 07, 053 (2020).

[10] X. H. Jin, Y. X. Gao, and D. J. Liu, Int. J. Mod. Phys. D 29 (2020).

[11] K. Jusufi, A. Banerjee, and S. G. Ghosh, Eur. Phys. J. C 80, 698 (2020). 
[12] M. Guo and P. Li, Eur. Phys. J. C 80, 588 (2020).

[13] C. Zhang, S. Zhang, P. Li, and M. Guo, JHEP 08, 105 (2020).

[14] S. Odintsov, V. Oikonomou, and F. Fronimos, Nucl. Phys. B 958, 115135 (2020).

[15] W. Ai, Commun. Theor. Phys. 72, 095402 (2020).

[16] P. G. Fernandes, P. Carrilho, T. Clifton, and D. J. Mulryne, Phys. Rev. D 102, 024025 (2020).

[17] R. A. Hennigar, D. Kubiznak, R. B. Mann, and C. Pollack, JHEP 2020, $27(2020)$.

[18] H. A. Gonzalez, M. Hassaine, C. Martinez, Phys. Rev. D 80, 104008 (2009).

[19] O. Miskovic, R. Olea, Phys. Rev. D 83, 024011 (2011).

[20] S. H. Hendi, S. Panahiyan, M. Momennia, Int. J. Mod. Phys. D 25, 1650063 (2016).

[21] D. Rubiera-Garcia, Phys .Rev. D 91, 064065 (2015).

[22] S. H. Hendi, B.Eslam, S. Panahiyan, Fortsch. Phys. 66, 1800005 (2018).

[23] S. Nojiri, S. D. Odintsov, Phys. Rev. D 96, 104008 (2017).

[24] C. H. Nam, Gen. Rel. Grav. 51, 104 (2019).

[25] S. Hyun, C. H. Nam, Eur. Phys. J. C 79, 737 (2019).

[26] M. S. Churilova, Z. Stuchlik, Ann. Phys. 418, 168181 (2020).

[27] K. Jusufi, Ann. Phys. 421, 168285 (2020).

[28] K. Jafarzade, M. K. Zangeneh, F. S. N. Lobo, Optical features of AdS black holes in the novel 4D Einstein-Gauss-Bonnet gravity coupled to nonlinear electrodynamics, arXiv:2009.12988 [gr-qc].

[29] S. I. Kruglov, Ann. Phys. (Berlin) 529, 1700073 (2017).

[30] S. W. Hawking and D. N. Page, Commun. Math. Phys. 87, 577 (1983). 
[31] Event Horizon Telescope collaboration, K. Akiyama et al., Astrophys. J. 875 (2019) L1; ibid L2; ibid L3; ibid L4; ibid L5; ibid L6.

[32] J. L. Synge, Mon. Not. Roy. Astron. Soc. 131, 463 (1966).

[33] M. Zhang and M. Guo, Eur. Phys. J. C 80, 790 (2020). 